\title{
Adsorptive Removal of Tetracyclines and Fluoroquinolones Using Yak Dung Biochar
}

\author{
Jun $\mathrm{Wu}^{1,2} \cdot$ Jian $\mathrm{Lu}^{3} \cdot$ Cui Zhang ${ }^{3} \cdot$ Zhenhua Zhang $^{4} \cdot$ Xiuyun Min $^{1,2}$
}

Received: 11 September 2018 / Accepted: 6 December 2018 / Published online: 14 December 2018

๑) Springer Science+Business Media, LLC, part of Springer Nature 2018

\begin{abstract}
This study investigated the adsorptive removal of target fluoroquinolones and tetracyclines by biochar made by yak dung collected from the Qinghai-Tibet Plateau. Yak dung biochar pyrolyzed at $700{ }^{\circ} \mathrm{C}$ possessed surface area of $198.745 \mathrm{~m}^{2} \mathrm{~g}^{-1}$ and multiple functional groups. Effects of different factors including biochar dosage, $\mathrm{pH}$, temperature, and initial concentration on adsorption of antibiotics were discussed. The biochar dosage of $1 \mathrm{~g} \mathrm{~L}^{-1}$ was preferable for adsorptive removal of antibiotics. Adsorption of target antibiotics did not show $\mathrm{pH}$-dependent features. Adsorption capacities of fluoroquinolones and tetracyclines positively increased with temperature and initial concentration. Pseudo-second order model and the Freundlich model better fitted the experimental kinetic and isotherm data, respectively. Yak dung biochar provides a new idea on resource utilization of solid wastes and pollution control in the Qinghai-Tibet Plateau.
\end{abstract}

Keywords Yak dung biochar $\cdot$ Adsorption $\cdot$ Antibiotics $\cdot$ Kinetics and isotherms

Biochar, a kind of carbonaceous functional material derived from biomass under the anaerobic or hypoxic conditions, has been widely used for removing various pollutants, amending soils, and sequestrating carbon (Kambo and Dutta 2015; Matovic 2011; Peiris et al. 2017; Shimabuku et al. 2016; Srinivasan and Sarmah 2015). Biochar generally possesses multiple functional groups such as $-\mathrm{COOH}$, alcoholic/phenolic-OH, and -CHO to show excellent capacity of removing toxic metals or organic pollutants (Wei et al. 2018). Meanwhile, biochar has also exhibited diverse advantages for application (Kambo and Dutta 2015; Peiris et al. 2017;

Jian Lu

jlu@yic.ac.cn

1 Key Laboratory of Comprehensive and Highly Efficient Utilization of Salt Lake Resources, Qinghai Institute of Salt Lakes, Chinese Academy of Sciences, Xining 810008, Qinghai, China

2 Qinghai Provincial Key Laboratory of Geology and Environment of Salt Lakes, 810008 Xining, Qinghai, China

3 Key Laboratory of Coastal Environmental Processes and Ecological Remediation, Yantai Institute of Coastal Zone Research, Chinese Academy of Sciences, 264003 Yantai, Shandong, China

4 School of Resources and Environmental Engineering, Ludong University, Yantai 264025, Shandong, China
Shimabuku et al. 2016; Wei et al. 2018). Therefore, biochar has become an effective, low-cost, and promising functional material for removing pollutants from various aquatic environments (Peiris et al. 2017; Shimabuku et al. 2016; Wei et al. 2018).

Recently, animal manure has been increasingly used to make biochar (Cao et al. 2011; Cely et al. 2015; Subedi et al. 2016) although diverse raw materials can be used for preparing biochar (Kambo and Dutta 2015; Srinivasan and Sarmah 2015; Wei et al. 2018). Yak dung is a kind of unique bio-waste in the Qinghai-Tibet Plateau to undertake multiple functions (Rhode et al. 2007; Yu et al. 2013). There are about 14 million yaks living in the Qinghai-Tibet Plateau (Yu et al. 2013). Approximately 16.1-26.8 million tons of yak dung is estimated to be excreted annually in the QinghaiTibet Plateau considering that amount of feces excreted by a yak is about 1150-1916 kg per year (Rhode et al. 2007). Therefore, effective utilization of yak dung becomes a challenge for sustainability of the Qinghai-Tibet Plateau and biochar is a promising utilization choice for yak dung.

Antibiotics have been extensively used to prevent/treat bacterial infections and promote yields of animal husbandry (Lu et al. 2018; Zhang et al. 2015). Fluoroquinolones, sulfonamides, macrolides, and tetracyclines account for approximately $55 \%$ of total antibiotic usage in China that is the largest antibiotic consumer in the world (Zhang 
et al. 2015). Moreover, antibiotics widely exist in various aquatic environments, especially wastewater (Lu et al. 2018; Shimabuku et al. 2016) to make removal of antibiotics from water critical to safety of aquatic environment. This study investigated the adsorptive removal of typical antibiotics using biochar pyrolyzed from yak dung. The final aim of this study is to provide important information on a low-cost and environment-friendly approach of controlling antibiotics in waters as well as utilization of yak dung in the QinghaiTibet Plateau.

\section{Materials and Methods}

Fluoroquinolone standards included ciprofloxacin (CFC) and enrofloxacin (EFC) while tetracycline standards comprised tetracycline (TC) and oxytetracycline (OTC). All antibiotic standards with purity of $98 \%$ were purchased from Sinopharm Chemical Reagent Co. (Shanghai, China). EFC, TC, and OTC were dissolved individually in methanol while CFC stock solution was prepared by dissolving CFC in $1 \%(\mathrm{v} / \mathrm{v})$ formic acid/methanol solution. All fresh antibiotic stock solutions with concentration of $100 \mathrm{mg} \mathrm{L}^{-1}$ were prepared biweekly and stored at $-20{ }^{\circ} \mathrm{C}$. Working solution was prepared before each assay by diluting the stock solution using methanol. HPLC-grade methanol and acetonitrile were purchased from Tedia Company (Fairfield, OH, USA).

Yak dung was collected from a yak breeding pasture located at Menyuan County of Qinghai Province, China. Yak dung was air dried under room temperature, smashed by a crusher to pass through $0.425 \mathrm{~mm}$ sieve, and put into a $100 \mathrm{~mL}$ crucible. The crucible was densely filled with yak dung, covered by a lid, and placed into a KSL-1200X muffle furnace (Hefei Ke Jing Materials Technology Co., Hefei, China). Heating rate of biochar preparation was set as $10{ }^{\circ} \mathrm{C} \mathrm{min}{ }^{-1}$. Biochars were produced at an operating temperature of $700^{\circ} \mathrm{C}$ with residence time of $2 \mathrm{~h}$.

The prepared biochars were homogenized and grounded to pass through a $0.075 \mathrm{~mm}$ sieve, and then dried at $105{ }^{\circ} \mathrm{C}$ for $12 \mathrm{~h}$ before characterization and batch assays. A CHN analyzer (Elementar micro cube, Germany) was used to analyze contents of $\mathrm{C}, \mathrm{H}$, and $\mathrm{N}$ of biochar. The $\mathrm{pH}$ of biochar was determined by measuring $\mathrm{pH}$ of the supernatants (biochar-water ratio of 1:2.5) using a pH meter (Shanghai INESA Scientific Instrument Co., China). Surface area of biochar was determined by fitting Brunauer, Emmett and Teller (BET) equation to nitrogen adsorption at $77 \mathrm{~K}$. Scanning electron microscope (SEM JSM-5610LV, JEOL, Japan) was used to investigate the surface morphology of biochar. Fourier transform infrared (FTIR) spectra of biochar were obtained by Nexus (Thermo Nicolet Corporation, USA).

Except the study exploring the effect of initial concentration on adsorption, the initial concentration of target antibiotics in the remaining experiments was $10 \mathrm{mg} \mathrm{L}^{-1}$. The adsorption experiments followed the below protocols if no specific conditions were pointed out. Biochar $(80 \mathrm{mg})$ was placed in a conical flask $(100 \mathrm{~mL})$ and $80 \mathrm{~mL}$ of synthetic wastewater was added. The flasks were tightly sealed by Parafilm and shaken at $180 \mathrm{rpm}$ under $25^{\circ} \mathrm{C}(298 \mathrm{~K})$ for $24 \mathrm{~h}$. Then biochar was separated from solution by centrifugation at $12000 \mathrm{rpm}$ for $5 \mathrm{~min}$. The supernatant was ready for ultra performance liquid chromatography (UPLC) analysis. All experiments were in triplicate. To discuss the effect of adsorbent dosage on adsorption, amounts of biochar were set as $0,2,4,8,16,40$, and $80 \mathrm{mg}$ to make biochar dosages of $0,0.025,0.05,0.1,0.2,0.5$, and $1 \mathrm{~g} \mathrm{~L}^{-1}$, respectively. To investigate the effect of $\mathrm{pH}$ on adsorption, solution was prepared at $\mathrm{pH}$ of 2-12. To explore impact of initial concentration on adsorption, initial concentrations of EFC and CFC were set as $1,5,10,20$, and $50 \mathrm{mg} \mathrm{L}^{-1}$ while those of TC were $1,5,10,20,50$ and $100 \mathrm{mg} \mathrm{L}^{-1}$ and those of OTC were 5, 10, 20, 50 and $100 \mathrm{mg} \mathrm{L}^{-1}$. Adsorptive kinetics of target antibiotics were performed at $25^{\circ} \mathrm{C}(298 \mathrm{~K})$, biochar dosage of $1 \mathrm{~g} \mathrm{~L}^{-1}$, and initial antibiotic concentration of $10 \mathrm{mg} \mathrm{L}^{-1}$ with sampling times of $0,0.167,0.5,1,2,3,4$, $5,6,8,10,12$, and $24 \mathrm{~h}$ for TC, EFC, and CFC. For OTC, water sample at $36 \mathrm{~h}$ was also taken. Isotherm experiments were performed under temperatures of $15{ }^{\circ} \mathrm{C}(288 \mathrm{~K}), 25^{\circ} \mathrm{C}$ $(298 \mathrm{~K})$, and $35^{\circ} \mathrm{C}(308 \mathrm{~K})$ with different concentrations ranging from 1 to $50 \mathrm{mg} \mathrm{L}^{-1}$ for $\mathrm{EFC}$ and $\mathrm{CFC}$ as well as $1-100 \mathrm{mg} \mathrm{L}^{-1}$ for TC and OTC.

Adsorptive kinetics of target antibiotics were described by pseudo-first order and pseudo-second order models according to Hameed and Daud (2008). The Langmuir model and Freundlich isotherm were used by this study (Hameed and Daud 2008; Yao et al. 2013) and briefly listed below:

$\frac{1}{q_{e q}}=\frac{1}{k_{L} Q_{m} C_{e q}}+\frac{1}{Q_{m}} ; q_{e q}=k_{F} C_{e q}^{\frac{1}{n}}$

where $q_{e q}$ and $C_{e q}$ are equilibrium amount of target antibiotics adsorbed by unit mass adsorbent and equilibrium concentration, respectively; $k_{L}$ is the surface adsorption equilibrium constant; $Q_{m}$ is the adsorption capacity under monolayer adsorption; $k_{F}$ is the Freundlich relative capacity coefficient; $n$ is the Freundlich empirical adsorption intensity coefficient.

Concentrations of target antibiotics were analyzed on an ACQUITY UPLC H-Class (Waters Corporation, Milford, USA). Chromatographic separation was performed on a reversed-phase C-18 column $(2.1 \mathrm{~mm} \times 50 \mathrm{~mm}, 1.7 \mu \mathrm{m}$ particle size) obtained from Waters Corporation (Milford, MA, USA) at a constant temperature of $40^{\circ} \mathrm{C}$. Mobile phase was $0.02 \mathrm{M}$ phosphate buffered saline (PBS) and acetonitrile with ratio of $85: 15(\mathrm{v}: \mathrm{v})$. The flow rate was $0.3 \mathrm{~mL} \mathrm{~min}^{-1}$ and sample injection volume was $10 \mu \mathrm{L}$. TC and OTC were 
detected at $355 \mathrm{~nm}$ while $\mathrm{CFC}$ and EFC were detected at $278 \mathrm{~nm}$.

Differences in removal rates and adsorption capacities of biochar for different target antibiotics were determined by ANOVA $(P=0.01)$.

\section{Results and Discussion}

The $\mathrm{pH}$ of biochar was 10.52 , similar to that reported by Subedi et al. (2016) but higher than that obtained by Cao et al. (2011). The contents of C, H, and $\mathrm{N}$ of yak dung biochar were $40.425 \%, 0.795 \%$, and $1.375 \%$, respectively. The Carbon content of yak dung biochar was lower than that of the previous reports (Cao et al. 2011; Subedi et al. 2016). Surface area of biochar was $198.745 \mathrm{~m}^{2} \mathrm{~g}^{-1}$ based on BET analysis, higher than that previous reported (Cao et al. 2011; Subedi et al. 2016). Biochar exhibited multirod like shape with porous structure based on the surface morphology (Fig. 1a). The absorption bands of biochar observed by FTIR spectra (Fig. 1b) represented the existence of some functional groups including $-\mathrm{OH}\left(3445 \mathrm{~cm}^{-1}\right)$, aromatic $\mathrm{C}=\mathrm{C} / \mathrm{C}=\mathrm{O}\left(1562 \mathrm{~cm}^{-1}\right), \mathrm{CH}_{2}-\left(1415 \mathrm{~cm}^{-1}\right), \mathrm{C}-\mathrm{O}$ $\left(1068 \mathrm{~cm}^{-1}\right)$, aromatic $\mathrm{CH}\left(875 \mathrm{~cm}^{-1}\right)$, etc (Bekiaris et al. 2016; Kumar et al. 2011; Liu et al. 2018). Considering previous report on application of biochar (Wei et al. 2018), the physico-chemical properties shown by this study make yak dung biochar possess potential of removing pollutants including antibiotics from waters.

It is a challenge to effectively treat antibiotics-containing waters such as agricultural runoff, aquaculture tail water, and soil solution. Therefore, removal of antibiotics using yak dung biochar would provide new insights for pollution control of antibiotics. Removal rates of target antibiotics by yak dung biochar increased with biochar dosage (Fig. 2a, b), reaching 77.78-91.14\% under biochar dosage of $1 \mathrm{~g} \mathrm{~L}^{-1}$. Removal rates of TC were higher than those of OTC when biochar dosage was $0.1 \mathrm{~g} \mathrm{~L}^{-1}$ or higher. Meanwhile, removal rates of CFC were also significantly higher than those of EFC when biochar dosage was $0.1 \mathrm{~g} \mathrm{~L}^{-1}$ or higher based on ANOVA analysis. Dosage of $1 \mathrm{~g} \mathrm{~L}^{-1}$ was acceptable and reasonable for adsorptive removal of fluoroquinolones and tetracyclines if considering the treatment cost.

Effect of $\mathrm{pH}$ on adsorption of target antibiotics was not significant when $\mathrm{pH}$ ranged from 6 to 10 although adsorption capacity of biochar for TC at $\mathrm{pH}$ of 6-10 was significantly higher than that for OTC and adsorption capacity of biochar for $\mathrm{CFC}$ at $\mathrm{pH}$ of 6-10 was remarkably higher than that for EFC based on ANOVA analysis (Fig. 2c, d). Interestingly, adsorption capacities of biochar for target antibiotics reached the highest at $\mathrm{pH}$ of 2. Adsorption capacities of biochar for TC and OTC showed different variation patterns. Adsorption capacity of biochar for OTC at $\mathrm{pH}$ of 12 was significantly higher than that at $\mathrm{pH}$ of 10 while adsorption capacity of biochar for $\mathrm{TC}$ at $\mathrm{pH}$ of 12 remarkably decreased. In contrast, adsorption capacities of biochar for EFC and CFC showed identical variation patterns similar with that for TC. The $\mathrm{pH}$ could affect the ionization state of the target antibiotics as well as biochar surface (Aghababaei et al. 2017). Target antibiotics in wastewater showed various forms such as molecule and ionic species under different $\mathrm{pH}$ while surface charges of biochar changed with pH (Aghababaei et al. 2017). Therefore, complex variation in adsorption capacities of biochar for fluoroquinolones and tetracyclines under different $\mathrm{pH}$ values showed $\mathrm{pH}$ might cause diverse impacts

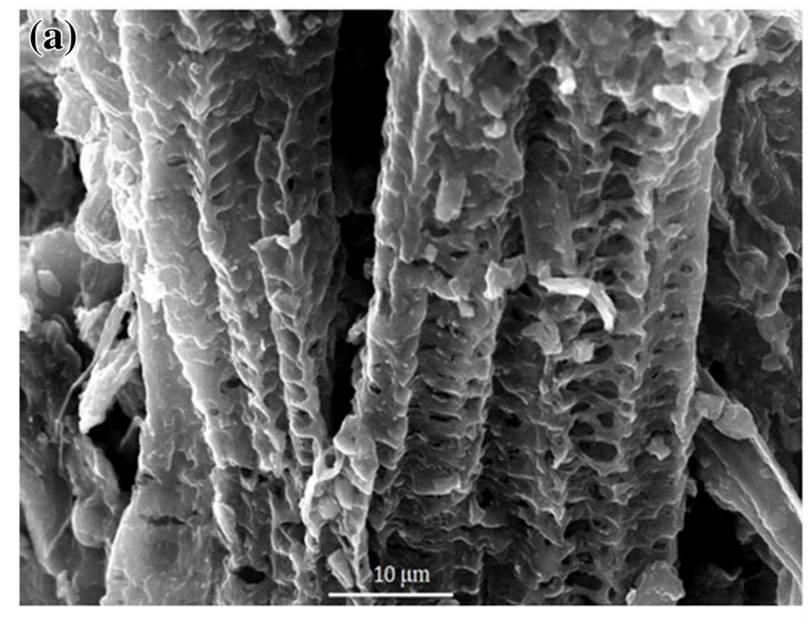

Fig. 1 SEM image (a) and FRIT spectrum (b) of yak dung biochar

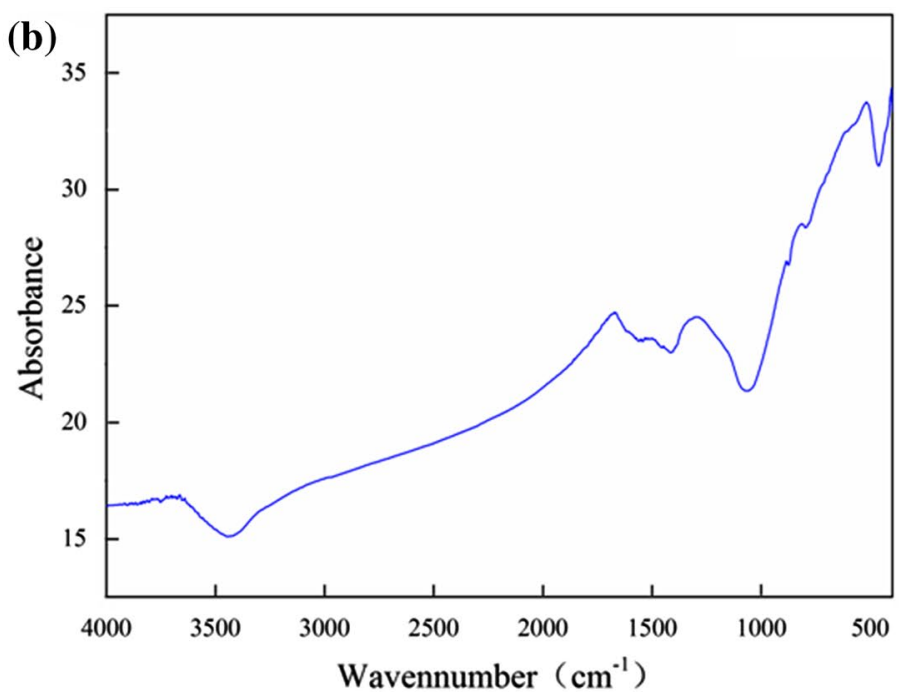



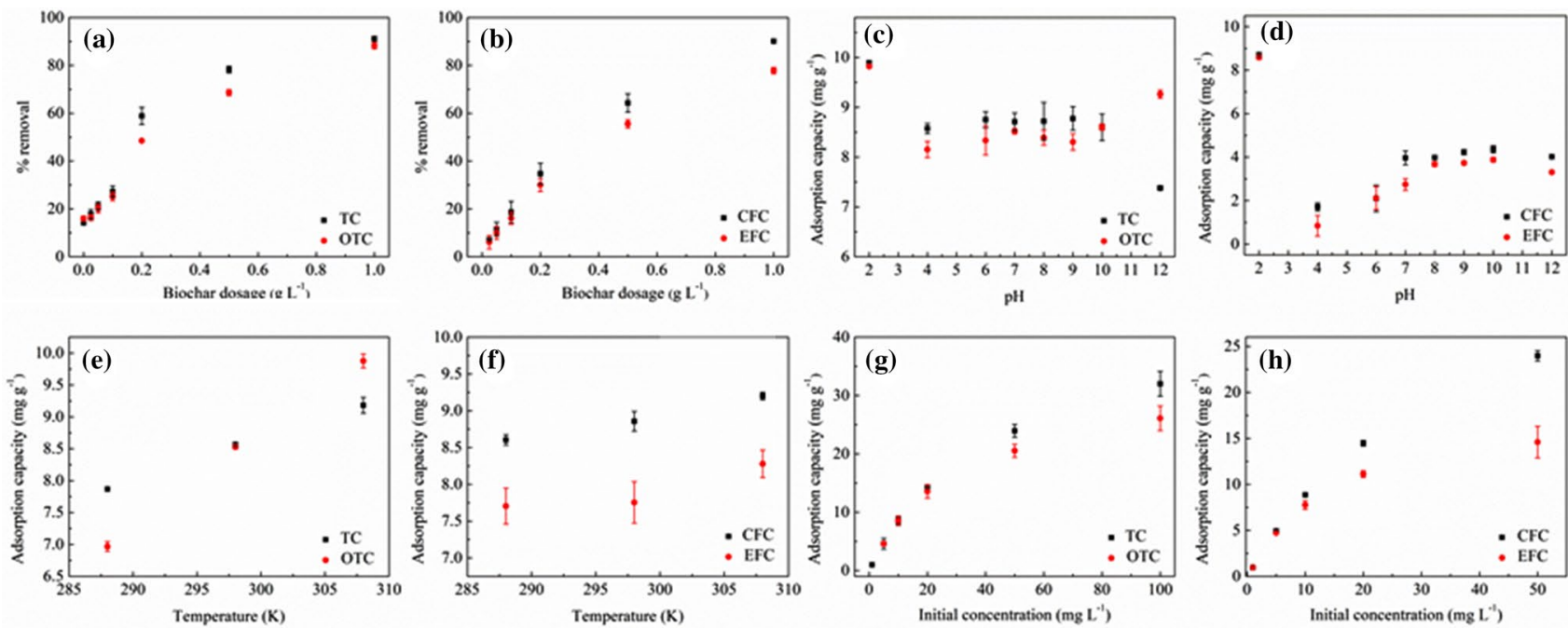

Fig. 2 Effects of biochar dosage $(\mathbf{a}, \mathbf{b}), \mathrm{pH}(\mathbf{c}, \mathbf{d})$, temperature $(\mathbf{e}, \mathbf{f})$, and initial concentration $(\mathbf{g}, \mathbf{h})$ on adsorption of target antibiotics by yak dung biochar

on adsorption. Moreover, the main influence mechanism of $\mathrm{pH}$ on adsorption of antibiotics using yak dung biochar still needed further exploration.

Effects of temperature on adsorption capacities of biochar for tetracyclines were more remarkable than those for fluoroquinolones (Fig. 2e, f). Adsorption capacities of biochar for target antibiotics increased with temperature, similar with the previous report (Aghababaei et al. 2017). Adsorption capacities of biochar for TC, OTC, EFC, and $\mathrm{CFC}$ at $35^{\circ} \mathrm{C}$ $(308 \mathrm{~K})$ were $1.17,1.42,1.07$, and 1.07 times those at $15^{\circ} \mathrm{C}$, respectively. Increase in temperature would be helpful to improve the effective collision between antibiotics and yak dung biochar as well as to enhance the number of active sites of biochar. Temperature had limited effects on adsorption of EFC by biochar, which might be related with the structure of EFC and its forms in aquatic phase.

Adsorption capacities of biochar for target antibiotics increased with initial concentrations of antibiotics (Fig. 2g, h), similar with the previous report (Wang et al. 2015). Initial concentration of antibiotics might affect the saturation time and adsorption equilibrium. Adsorption capacity of biochar for TC with the initial concentration of $100 \mathrm{mg} \mathrm{L}^{-1}$ was 32.75 times that with the initial concentration of $1 \mathrm{mg} \mathrm{L}^{-1}$, reaching $32.01 \mathrm{mg} \mathrm{g}^{-1}$. Adsorption capacity of biochar for OTC with the initial concentration of $100 \mathrm{mg} \mathrm{L}^{-1}$ was 5.67 times that with the initial concentration of $5 \mathrm{mg} \mathrm{L}^{-1}$, reaching $26.09 \mathrm{mg} \mathrm{g}^{-1}$. Effect of the initial concentration on adsorption capacity of biochar for CFC was more remarkable than that for EFC. Adsorption capacities of biochar for CFC and EFC with the initial concentration of $1 \mathrm{mg} \mathrm{L}^{-1}$ were 0.95 and $0.98 \mathrm{mg} \mathrm{g}^{-1}$, respectively while those for CFC and EFC with the initial concentration of $50 \mathrm{mg} \mathrm{L}^{-1}$ were 23.97 and $14.59 \mathrm{mg} \mathrm{g}^{-1}$, respectively.
Pseudo-first order and pseudo-second order models were used to discuss adsorption processes of target antibiotics on yak dung biochar (Fig. 3). Biochar adsorption of TC reached equilibrium faster than that of OTC. Desorption rates of OTC and TC on biochar were less than $0.7 \%$ within $72 \mathrm{~h}$. Biochar adsorption of EFC reached equilibrium faster than that of CFC. Desorption rates of EFC and CFC on biochar were less than $0.5 \%$ within $72 \mathrm{~h}$. Pseudo-second order model fitted better with the experimental data for all target antibiotics than pseudo-first order model when comparing the correlation coefficients $\left(\mathrm{R}^{2}\right)$. The rate constants $\left(k_{2}\right)$ for pseudo-second-order model of all target antibiotics ranged from 0.31 (CFC) to 2.76 (TC). Parameter $k_{2}$ values of tetracyclines were higher than those of fluoroquinolones. The calculated equilibrium adsorption capacities of biochar for all target antibiotics were similar.

The Langmuir and Freundlich models were used to investigate adsorption isotherm of target antibiotics on yak dung biochar (Fig. 4). Adsorption of TC, EFC, and CFC on biochar did not follow the Langmuir isotherm while the Freundlich isotherm fitted well with adsorption data of these antibiotics. The Freundlich isotherm yielded better fitting results for adsorption of OTC than the Langmuir isotherm. The Freundlich isotherm fitted well with adsorption data of target antibiotics, possibly showing that the adsorption of fluoroquinolones and tetracyclines did not occur at specific homogeneous sites of yak dung biochar (Yao et al. 2013). The Freundlich relative capacity coefficients $\left(k_{F}\right)$ of all target antibiotics increased with temperature. The highest $k_{F}$ values of TC, OTC, EFC, and CFC at $35^{\circ} \mathrm{C}(308 \mathrm{~K})$ reached 9.82 , $13.16,6.51$, and 8.89 , being $1.47,2.64,1.11$, and 1.25 times those at $15^{\circ} \mathrm{C}(288 \mathrm{~K})$. Adsorption of OTC was more sensitive to temperature than the other antibiotics. 

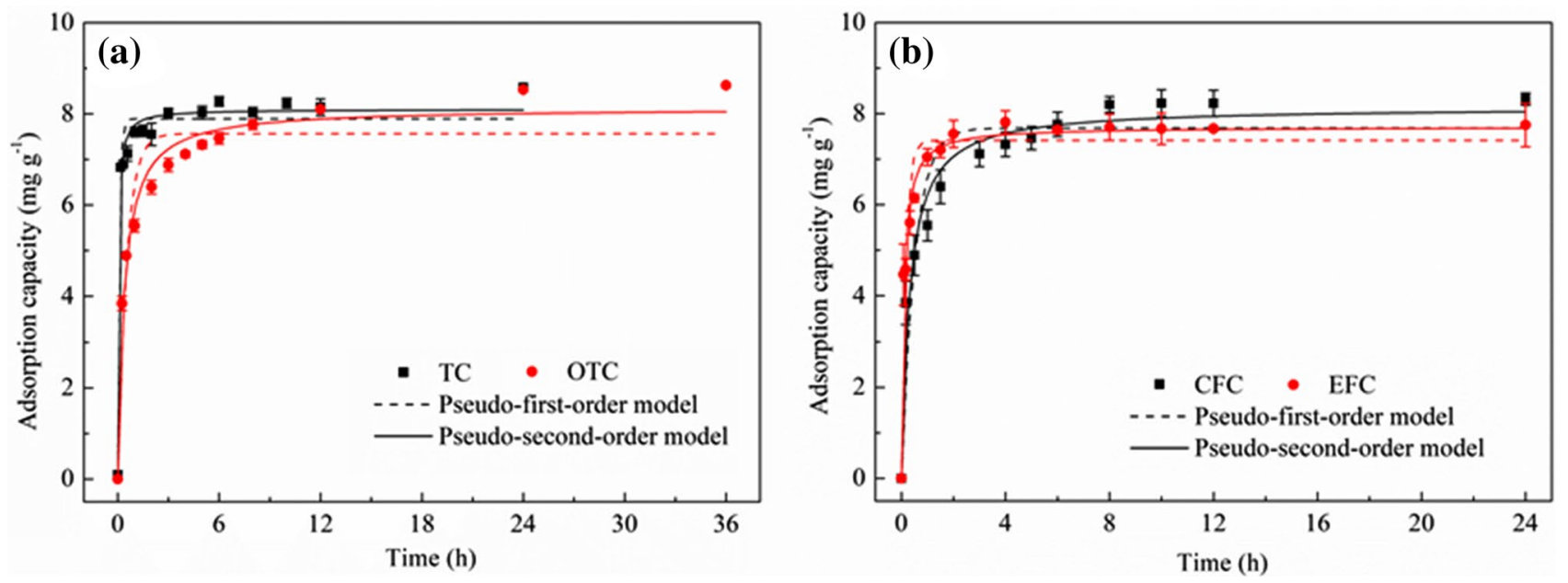

Fig. 3 Adsorption kinetics of target tetracyclines (a) and fluoroquinolones (b) on yak dung biochar
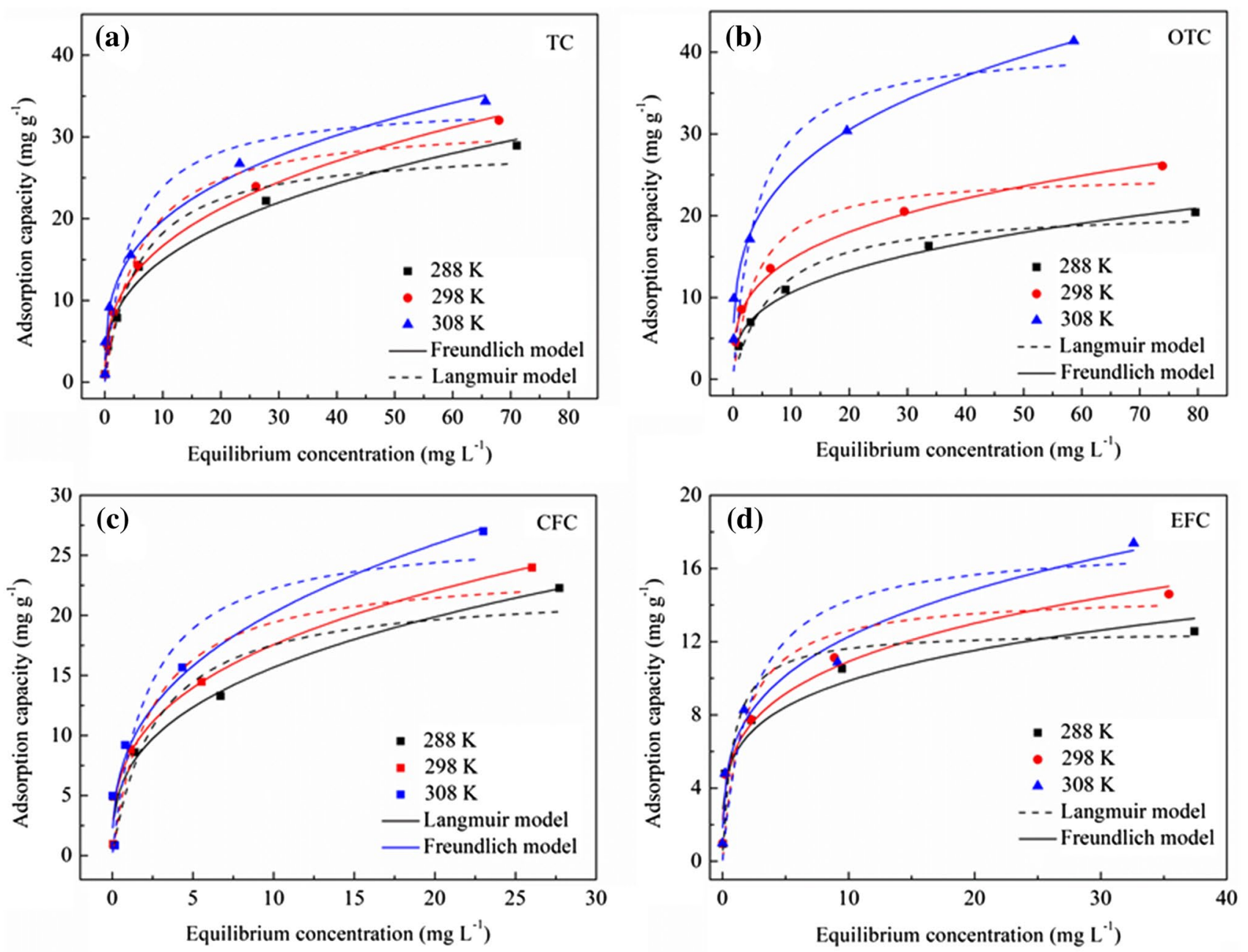

Fig. 4 Adsorption isotherm of tetracycline (a), oxytetracycline (b), ciprofloxacin (c), and enrofloxacin (d) on yak dung biochar 
The disposal of biochar utilized for adsorbing antibiotics is important for environmental safety and sustainability. Currently, incineration and biochar re-preparation might be two feasible approaches for treating biochar after utilization. However, it is still necessary to develop more environmental-friendly disposal methods in the future.

Acknowledgements This work was supported by National Natural Science Foundation of China (Grant Nos. 41671319 and 41877131), One Hundred Talents Program of Chinese Academy of Sciences (Grant numbers of Y610061033 and Y629041021), Thousand Talents Plan of Qinghai Province (Grant No. Y740171071), Taishan Scholar Program of Shandong Province, Two-Hundred Talents Plan of Yantai (Grant No. Y739011021), Natural Science Foundation of Qinghai Province (Grant No. 2019-ZJ-909), and Applied Basic Research Program of Qinghai Province (Grant No. 2016-ZJ-755). The authors would like to thank the reviewers for their valuable suggestions and comments on the manuscript.

\section{References}

Aghababaei A, Ncibi MC, Sillanpää M (2017) Optimized removal of oxytetracycline and cadmium from contaminated waters using chemically-activated and pyrolyzed biochars from forest and wood-processing residues. Bioresour Technol 239:28-36

Bekiaris G, Peltre C, Jensen LS, Bruun S (2016) Using FTIR-photoacoustic spectroscopy for phosphorus speciation analysis of biochars. Spectrochim Acta A 168:29-36

Cao X, Ma L, Liang Y, Gao B, Harris W (2011) Simultaneous immobilization of lead and atrazine in contaminated soils using dairymanure biochar. Environ Sci Technol 2011 45:4884-4889

Cely P, Gascó G, Paz-Ferreiro J, Méndez A (2015) Agronomic properties of biochars from different manure wastes. J Anal Appl Pyrolysis 111:173-182

Hameed BH, Daud FBM (2008) Adsorption studies of basic dye on activated carbon derived from agricultural waste: hevea brasiliensis seed coat. Chem Eng J 139:48-55

Kambo HS, Dutta A (2015) A comparative review of biochar and hydrochar in terms of production, physico-chemical properties and applications. Renew Sustain Energy Rev 45:359-378

Kumar S, Loganathan VA, Gupta RB, Barnett MO (2011) An Assessment of $\mathrm{U}(\mathrm{VI})$ removal from groundwater using biochar produced from hydrothermal carbonization. J Environ Manag 92:2504-2512

Liu S, Liu Y, Tan X, Zeng G, Zhou Y, Liu S, Yin Z, Jiang L, Li M, Wen J (2018) The effect of several activated biochars on Cd immobilization and microbial community composition during in-situ remediation of heavy metal contaminated sediment. Chemosphere 208:655-664

Lu J, Wu J, Zhang C, Zhang Y, Lin Y, Luo Y (2018) Occurrence, Distribution, and Ecological-Health Risks of Selected Antibiotics in Coastal Waters along the Coastline of China. Sci Total Environ 644:1469-1476

Matovic D (2011) Biochar as a viable carbon sequestration option: Global and Canadian perspective. Energy 36:2011-2016

Peiris C, Gunatilake SR, Mlsna TE, Mohan D, Vithanage M (2017) Biochar based removal of antibiotic sulfonamides and tetracyclines in aquatic environments: A critical review. Bioresour Technol 246:150-159

Rhode D, Madsen DB, Brantingham PJ, Dargye T (2007) Yaks, yak dung, and prehistoric human habitation of the Tibetan Plateau. Dev Quat Sci 9:205-224

Shimabuku KK, Kearns JP, Martinez JE, Mahoney RB, MorenoVasquez L, Summers RS (2016) Biochar sorbents for sulfamethoxazole removal from surface water, stormwater, and wastewater effluent. Water Res 96:236-245

Srinivasan P, Sarmah AK (2015) Characterisation of agricultural waste-derived biochars and their sorption potential for sulfamethoxazole in pasture soil: a spectroscopic investigation. Sci Total Environ 502:471-480

Subedi R, Taupe N, Ikoyi I, Bertora C, Zavattaro L, Schmalenberger A, Leahy JJ, Grignani C (2016) Chemically and biologicallymediated fertilizing value of manure-derived biochar. Sci Total Environ 550:924-933

Wang Y, Lu J, Wu J, Liu Q, Zhang H, Jin S (2015) Adsorptive removal of fluoroquinolone antibiotics using bamboo biochar. Sustainability 7:12947-12957

Wei D, Li B, Huang H, Luo L, Zhang J, Yang Y, Guo J, Tang L, Zeng G, Zhou Y (2018) Biochar-based functional materials in the purification of agricultural wastewater: fabrication, application and future research needs. Chemosphere 197:165-180

Yao H, Lu J, Wu J, Lu Z, Wilson PC, Shen Y (2013) Adsorption of fluoroquinolone antibiotics by wastewater sludge biochar: role of the sludge source. Water Air Soil Pollut 224:1370

Yu X, Xu C, Wang F, Shang Z, Long R (2013) Levels of germinable seed in topsoil and Yak Dung on an alpine meadow on the NorthEast Qinghai-Tibetan plateau. J Integr Agric 12(12):2243-2249

Zhang Q-Q, Ying G-G, Pan C-G, Liu Y-S, Zhao J-L (2015) Comprehensive evaluation of antibiotics emission and fate in the river basins of china: source analysis, multimedia modeling, and linkage to bacterial resistance. Environ Sci Technol 49:6772-6782 